\title{
Studies on the growth and characterization of $p$-hydroxyacetophenone single crystals
}

\author{
N. Vijayan ${ }^{\mathrm{a}}$, R. Ramesh Babu ${ }^{\mathrm{a}}, \mathrm{M}$. Gunasekaran $^{\mathrm{a}}, \mathrm{R}$. Gopalakrishnan $^{\mathrm{a}, \mathrm{d}, *}$, \\ R. Kumaresan ${ }^{\text {b }, ~ P . ~ R a m a s a m y ~}{ }^{\mathrm{c}}$, C.W. Lan ${ }^{\mathrm{d}}$ \\ ${ }^{a}$ Department of Physics, Anna University, Chennai 600 025, India \\ ${ }^{\mathrm{b}}$ Department of Electrical and Computer Engineering, Nagoya Institute of Technology, Gokiso-cho, Showaku, Nagoya 466, Japan \\ ${ }^{\mathrm{c}}$ Crystal Growth Centre, Anna University, Chennai 600 025, India \\ ${ }^{\mathrm{d}}$ Department of Chemical Engineering, National Taiwan University, Taipei 10617, Taiwan, ROC
}

Received 12 August 2002; accepted 22 August 2002

Communicated by M. Schieber

\begin{abstract}
Good-quality single crystals of organic $p$-hydroxyacetophenone have been grown by slow evaporation solution growth technique at $37^{\circ} \mathrm{C}$ using methanol as solvent. The grown crystals have been characterized by Raman studies, FT-IR, X-ray powder diffraction and thermal analyses. The mechanical properties of the grown crystals have been studied using Vicker's micro-hardness tester.
\end{abstract}

(C) 2002 Published by Elsevier Science B.V.

PACS: 81.10.Dn; 42.70.Mp; 81.07.-b; 81.70.Pg

Keywords: A1. Characterization; A1. Thermal gravimetry; A1. X-ray diffraction; A2. Growth from solutions; B1. Organic compounds

\section{Introduction}

Nonlinear optics (NLO) has wide applications in the field of telecommunication and optical information storage devices. The organic NLO materials play an important role in SHG, frequency mixing, electro-optic modulation, optical parametric oscillation, optical bi-stability, etc. [1]. Organic crystals have parameters superior to widely used crystals like KDP [2-4]. The organic

\footnotetext{
*Corresponding author. Department of Physics, Anna University, Chennai 600 025, India.

E-mail address: krgkrishnan@annauniv.edu

(R. Gopalakrishnan).
}

NLO materials have an order of magnitude higher second harmonic generation efficiencies and also exhibit substantially greater laser damage thresholds. Considerable work has been done in order to understand the microscopic origin of nonlinear behavior of organics $[5,6]$. The NLO properties of large organic molecules and polymers have been the subject of extensive theoretical and experimental investigations during the past two decades and they have been investigated widely due to their high nonlinear optical properties, rapid response in electrooptic effect and large second- or third-order hyperpolarizibilities compared to inorganic NLO materials [7]. 
Recently, a large number of organic compounds with nonlocalized $\pi$ electron systems and a large dipole moment have been synthesized to realize the nonlinear susceptibilities larger than the inorganic optical materials. However, their potential applications are limited by poor chemical stability, by red cut-off wavelength caused by a large organic nconjugated system and by poor phase matching properties caused by large birefringence which results from the layer stacking of the structure and by other factors [8]. The basic structure of organic NLO materials is based on $\pi$ bond systems. Due to the overlap of $\pi$ orbital, delocalization of electronic charge distribution leads to a high mobility of the electron density. Functionalization of both ends of the $\pi$ bond system with appropriate electron donor and acceptor groups can enhance the asymmetric electronic distribution in either or both the ground and excited states, these leading to an increased optical nonlinearity [9].

The low-temperature solution growth technique is widely used for the growth of organic and inorganic compounds to get good single crystals [10]. In this paper we present the growth details and analyses of $p$-hydroxyacetophenone. $p$-hydroxyacetophenone $\left(\mathrm{C}_{8} \mathrm{H}_{8} \mathrm{O}_{2}\right)$ is one of the potential organic NLO material. It has been grown by slow evaporation solution growth technique. The Raman spectrum of the 4-hydroxyacetophenone shows more intense absorption for powder sample compared to as-grown crystal. The $\mathrm{C}-\mathrm{H}$ bend is more intense in the crystal sample than in the powder sample.

\section{Experimental procedure}

\subsection{Crystal growth}

The $p$-hydroxyacetophenone belongs to $\mathrm{P} 2{ }_{1} 2_{1} 2_{1}$ space group and aromatic ketone class with a noncentrosymmetrical structure. The low-temperature solution has been employed to grow the above crystal using an alcoholic solvent as it is insoluble in water. The commercially available 4hydroxyacetophenone $(99.99 \%)$ has been purified by repeated recrystallization process using methanol as solvent. A very small amount of charcoal was added to the solution in order to remove the impurities. The recrystallization was done in order to improve the optical quality as well as the size of the growing crystals. It was found that the growth occurs in a faster manner in methanol compared to ethanol and hence methanol was used as a solvent for our growth.

The saturated solution of 4-hydroxyacetophenone was obtained by dissolving the recrystallized material with continuous stirring of the solution using a magnetic stirrer. On reaching saturation, the equilibrium concentration of the solute was determined by gravimetry. The beaker containing the solution was optimally closed for controlled evaporation. The solution was kept in a constant temperature bath at $37^{\circ} \mathrm{C}$. Transparent single crystals were obtained from the mother solution after 15 days (Fig. 1). The molecular structure of the grown crystal is given in Scheme 1 .

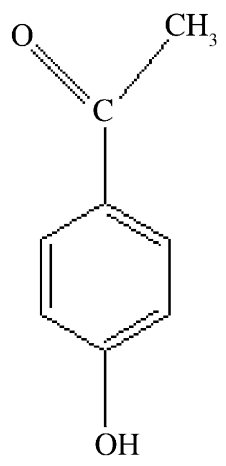

Scheme 1. Molecular structure of $p$-hydroxyacetophenone.

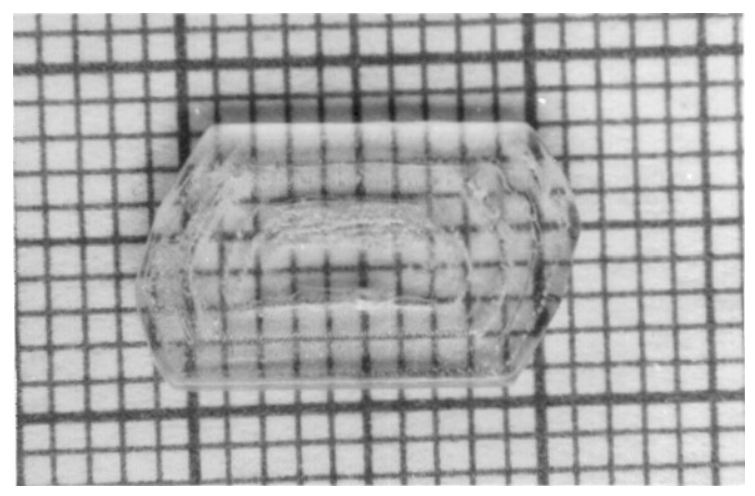

Fig. 1. Single crystal of $p$-hydroxyacetophenone (4-hydroxyacetophenone). 


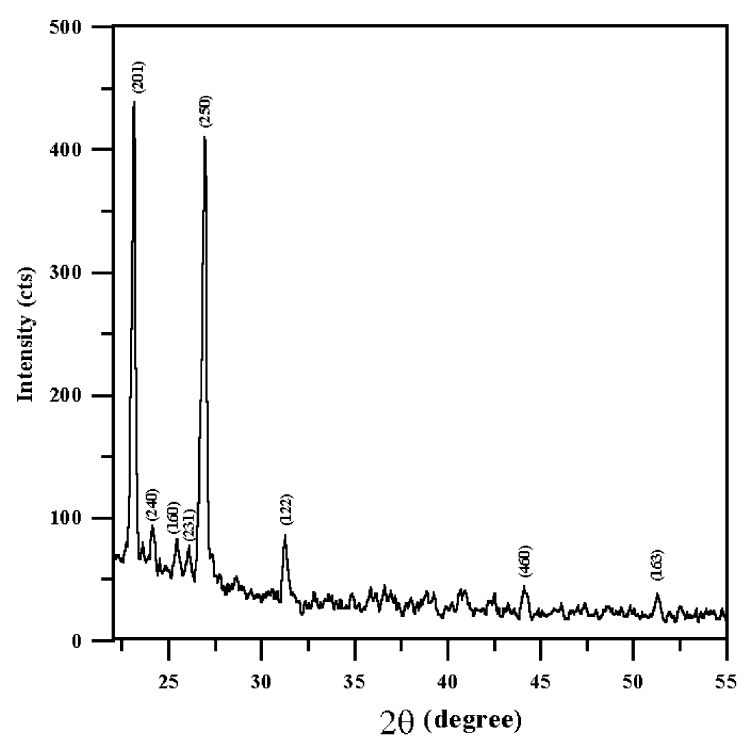

Fig. 2. Powder X-ray spectrum of $p$-hydroxyacetophenone.

\section{Crystal characterization}

\subsection{X-ray diffraction analysis}

The grown crystals have been characterized by $\mathrm{X}$-ray powder diffraction technique using the Rich Seifert powder X-ray diffractometer. From the powder X-ray diffraction data the lattice parameters and the cell volume have been calculated using the celn software package. The obtained results are in good agreement with the reported XRD values [6]. The peak corresponding to $(201)$ has a maximum count of 430 cts. This is the strongest diffraction peak. From the X-ray diffraction data we observe that it belongs to $\mathrm{P} 2{ }_{1} 2_{1} 2_{1}$ space group and orthorhombic crystal system. Fig. 2 represents the powder X-ray diffraction pattern of the grown $p$-hydroxyacetophenone single crystals. The crystallographic data are given in Table 1.

\subsection{Raman spectra analysis}

The Raman spectra of $p$-hydroxyacetophenone have been recorded between 200 and $2000 \mathrm{~cm}^{-1}$ both for single crystal and the powder form. Figs. $3 \mathrm{a}$ and $\mathrm{b}$ show the Raman spectra recorded
Table 1

Crystallographic data of $p$-hydroxyacetophenone

\begin{tabular}{lllll}
\hline$a$ & $b$ & $c$ & $\alpha=\beta=\gamma$ & Volume \\
\hline $9.541 \AA$ & $24.340 \AA$ & $6.132 \AA$ & $90^{\circ}$ & $1424 \AA^{3}$ \\
\hline
\end{tabular}

on the grown crystal and powdered sample, respectively. The spectrum shows more intense absorption for powder sample compared to asgrown crystal. In both spectra, the $\mathrm{C}=\mathrm{O}$ stretching mode yields less intense $1650 \mathrm{~cm}^{-1}$ and more intense $1595 \mathrm{~cm}^{-1}$ lines. The less intense peak is assigned to free $\mathrm{C}=\mathrm{O}$ and the more intense peak is assigned to hydrogen bonded $\mathrm{C}=\mathrm{O}$. As the hydrogen bonded $\mathrm{C}=\mathrm{O}$ absorption (lower wave number mode) is more intense than that of the free $\mathrm{C}=\mathrm{O}$ in the crystal, it is expected that most of the $\mathrm{C}=\mathrm{O}$ groups of $p$-hydroxyacetophenone in the crystal are hydrogen bonded [6]. When the intensities of these two bands of the crystal are compared with that of the powder sample, the latter sample is observed to have much more intensity than the former. This is to be attributed to more number of hydrogen bonded free $\mathrm{C}=\mathrm{O}$ 's in the powder sample compared to the crystal. Thus, powdering of the crystal reduces the bonding forces within the crystal and also between molecules. In addition to $\mathrm{C}=\mathrm{O}$, the skeletal vibrations $\left(1440,1520\right.$ and $1565 \mathrm{~cm}^{-1}$ ) of the aromatic ring are also found to produce more intense absorption in the powder sample than the crystal, illustrating more freedom for skeletal vibration in the powder than in the crystal. The bands between 1225 and $1500 \mathrm{~cm}^{-1}$ are assigned to $\mathrm{CH}_{3}$ symmetric bends. The phenolic $\mathrm{C}=\mathrm{O}$ stretching mode also provides two peaks between 1000 and $1225 \mathrm{~cm}^{-1}$ and these two bands are also more intense for powder than for the crystal. The aromatic $\mathrm{C}-\mathrm{H}$ bend produces intense peak in the crystal than in the powder sample.

\subsection{Thermal gravimetric analysis}

The thermal gravimetric analysis (TGA) of the sample was carried out in METTLER-TA4000 system, about $17 \mathrm{mg}$ of the sample was heated in a 

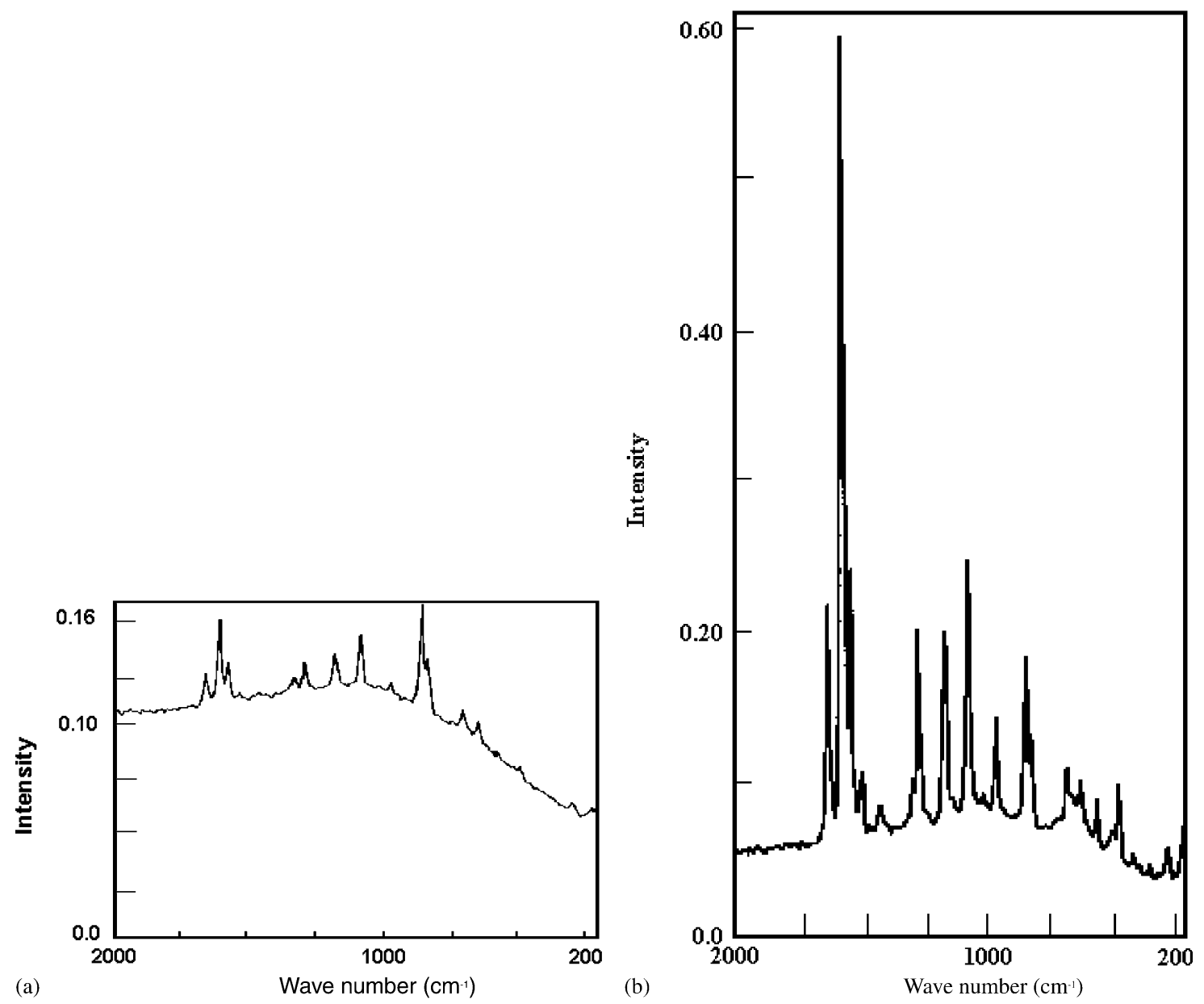

Fig. 3. (a) Raman spectrum of p-hydroxyacetophenone (crystal sample). (b) Raman spectrum of $p$-hydroxyacetophenone (powder sample).

crucible between $35^{\circ} \mathrm{C}$ and $700^{\circ} \mathrm{C}$ at a heating rate of $20^{\circ} \mathrm{C} / \mathrm{min}$ in air. The resulting thermogram and the DTG trace are illustrated in Fig. 4. There is a sharp single stage of weight loss between $140^{\circ} \mathrm{C}$ and $300^{\circ} \mathrm{C}$. There is no loss of water below $125^{\circ} \mathrm{C}$ illustrating the absence of any absorbed water in the sample. Since, the single stage of weight loss starts at $147^{\circ} \mathrm{C}$ without any intermediate stages the compound is expected to evaporate at this temperature. This study indicates that the compound could be used for any application below its melting point. The sharpness of the thermogram is also illustrative of the crystal purity without association of any impurities.

\subsection{FT-IR analysis}

The middle IR spectrum of $p$-hydroxyacetophenone was scanned between 400 and $4000 \mathrm{~cm}^{-1}$ by $\mathrm{KBr}$ technique (Model: BRUKER IFS 66 V FTIR). It is shown in Fig. 5. The higher wave number region gives interesting informations with regard to bonding of the groups in the crystal. The phenolic $\mathrm{OH}$ stretch provides sharp as well as broad peaks illustrating the presence of free $\mathrm{OH}$ grouping without intermolecular bonding and intensely hydrogen bonded $\mathrm{OH}$ groupings. The former free $\mathrm{OH}$ groupings provide their stretching patterns at $3483.84,3452.87$ and $3392.66 \mathrm{~cm}^{-1}$. 


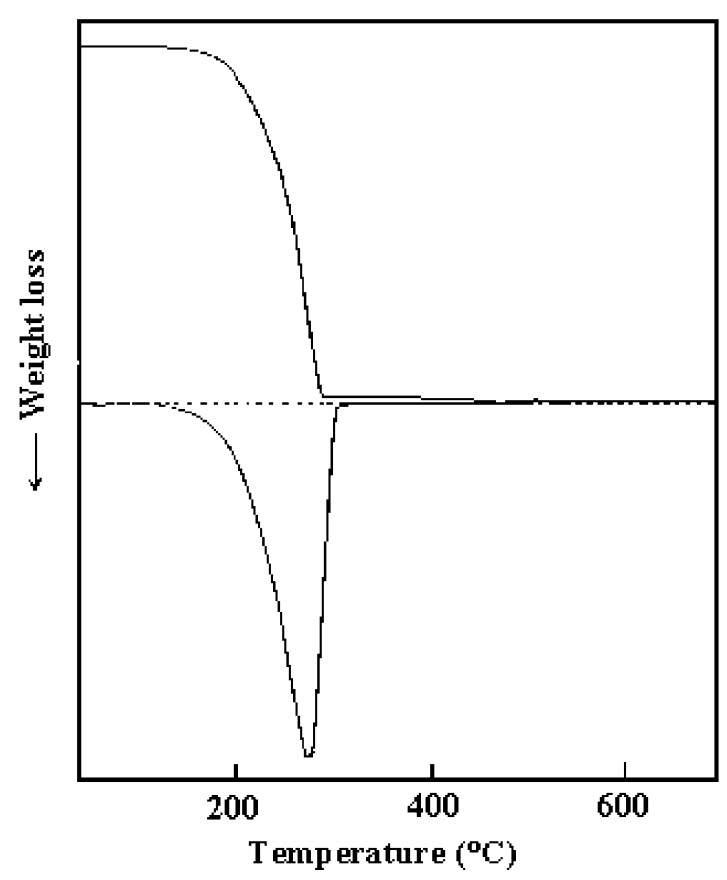

Fig. 4. TGA spectrum of $p$-hydroxyacetophenone.
The broad peak due to hydrogen bonded $\mathrm{OH}$ groupings has its peak maximum at 3205.97 and $3321.03 \mathrm{~cm}^{-1}$. The aromatic $\mathrm{CH}$ and aliphatic $\mathrm{CH}$ stretching modes are not clearly resolved even though their presence is clearly seen at 3010 and $2950 \mathrm{~cm}^{-1}$. The carbonyl $\mathrm{C}=\mathrm{O}$ stretch is observed at $1694.99 \mathrm{~cm}^{-1}$. Its appearance illustrates nearly same type of interaction between carbonyl groups of all molecules with the environment in the crystal. Hence in the crystal to bind the molecules together, the $\mathrm{C}=\mathrm{O}$ groups are entirely involved in bonding and $\mathrm{OH}$ groups are not fully involved in bonding. The skeletal vibrations of the aromatic ring are observed to produce peaks at 1603.43 , $1567.49,1516.01$ and $1463.03 \mathrm{~cm}^{-1}$. The peaks at 1367.8 and $1450 \mathrm{~cm}^{-1}$ indicate $\mathrm{CH}_{3}$ bending modes. Phenolic $\mathrm{C}=\mathrm{O}$ stretch is seen to produce a doublet at 1273.88 and $1310.34 \mathrm{~cm}^{-1}$ due to the presence of free and hydrogen bonded groupings. The para substitution is clearly evident by the peak at $832.08 \mathrm{~cm}^{-1}$. There is an intense peak at $1111.64 \mathrm{~cm}^{-1}$ which may be assigned to $-\mathrm{C}-\mathrm{CO}-$ $\mathrm{CH}_{3}$ stretch and bend of the constituent.

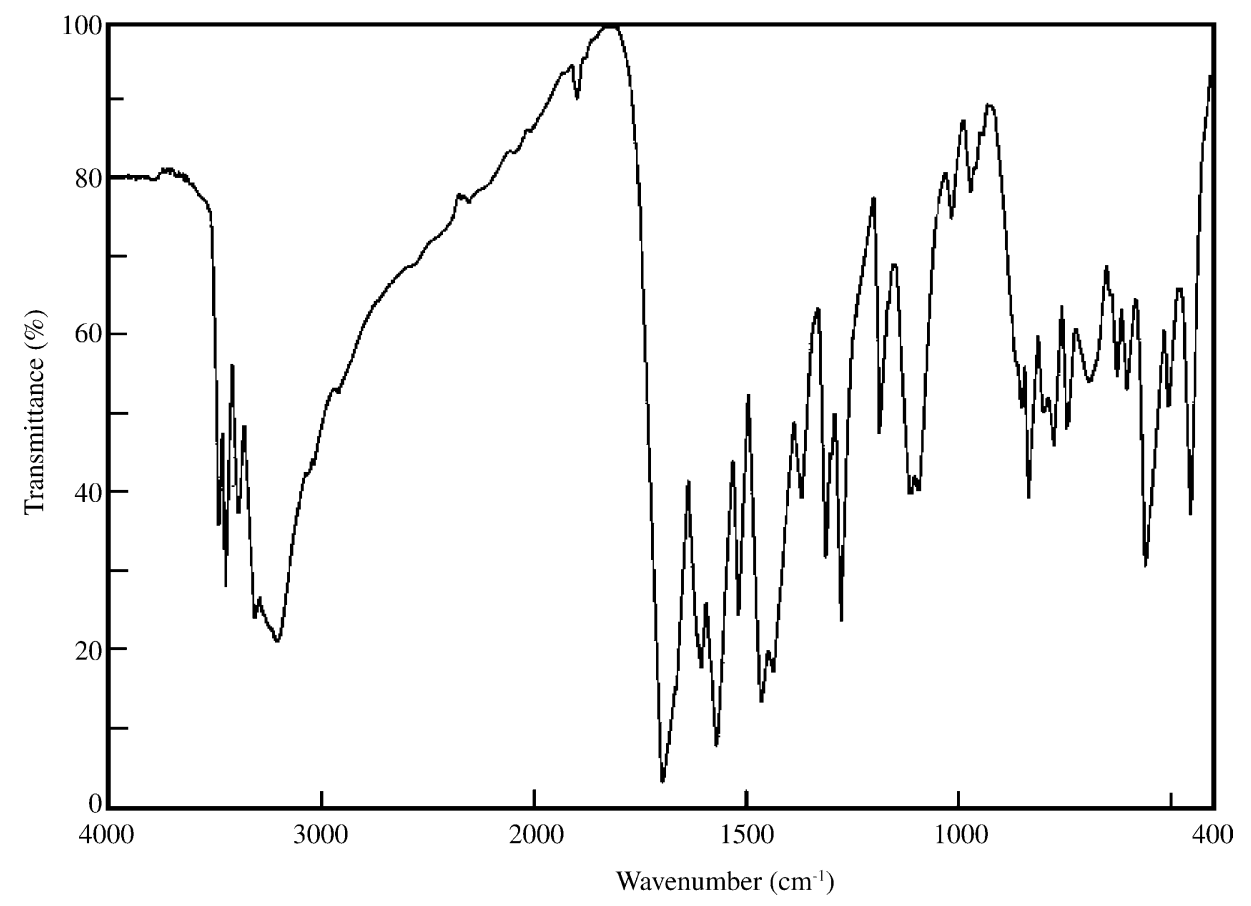

Fig. 5. FT-IR spectrum of $p$-hydroxyacetophenone. 


\subsection{Optical assessment}

The UV-Vis absorbance of the compound was scanned between 200 and $800 \mathrm{~nm}$ (Fig. 6) (Model: VARIAN CARRY 5E UV-Vis NIR). Generally, pure acetophenone absorbs at 246 and $280 \mathrm{~nm}$. Both are assigned to $\pi-\pi^{*}$ transition. In the $p$ hydroxyacetophenone, the absorbance maximum at $315 \mathrm{~nm}$ is to be assigned to $n-\pi^{*}$ transition of the constituent group. But the material is transparent to wavelengths ranging from $350-400 \mathrm{~nm}$ and the entire visible region. Hence, the material can be used for NLO applications. In order to confirm the NLO property, the grown crystal has been tested using Nd:YAG laser. When the $\mathrm{Nd}$ :YAG laser light was incident on the crystal sample the second harmonic generation is developed on the crystal samples (confirmed by emission of green radiation).

\subsection{Hardness studies}

Micro-hardness studies have been carried out on the 4-hydroxyacetophenone single crystals using a Leitz micro-hardness tester fitted with a Vickers diamond pyramidal indentor. Vickers microhardness values have been calculated using $H_{\mathrm{v}}=1.8544 P / d^{2} \mathrm{~kg} / \mathrm{mm}^{2}$ where $P$ is the applied load and $d$ is the mean diagonal length of the indentor impression. Hardness values have been taken for various applied loads.

A graph has been plotted between hardness number $\left(H_{\mathrm{v}}\right)$ and applied load $(P)$ (Fig. 7). It is observed from the graph that the hardness value

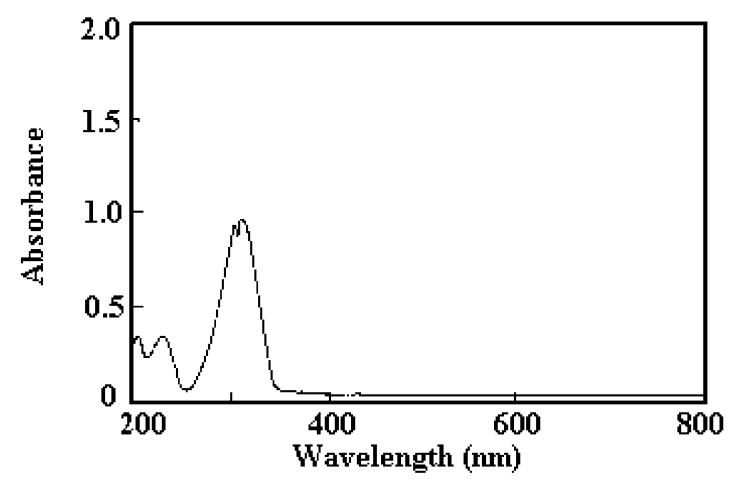

Fig. 6. UV-Vis spectrum of $p$-hydroxyacetophenone.

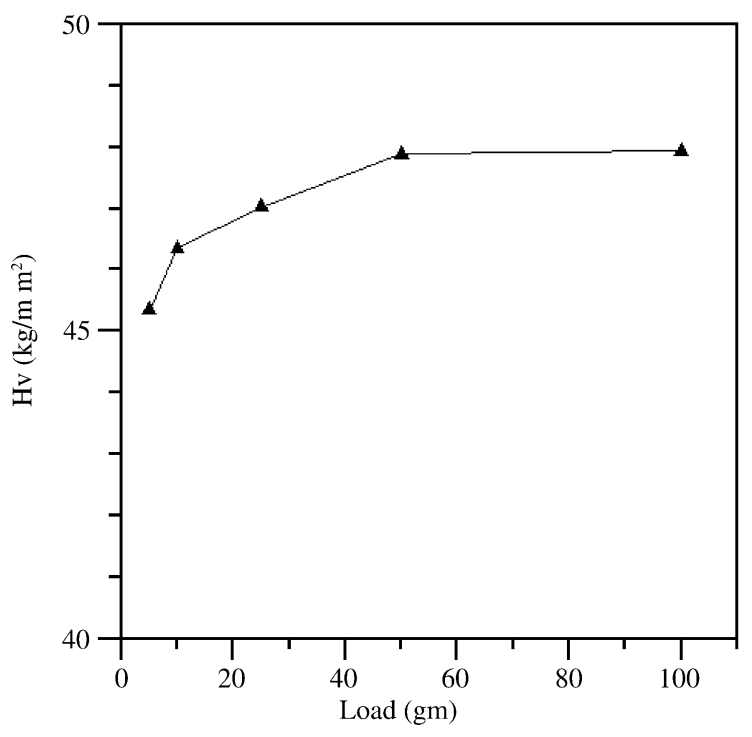

Fig. 7. Plot of load (P) vs. Vicker's hardness.

increases and then attains almost saturation with the increase of the applied load. A plot obtained between $\ln (P)$ against $\ln (d)$ gives a straight line, which is derived from the Meyer's law, the relation connecting the applied load is given by $P=a d^{n}$. Here, $n$ is the Meyer index or work hardening coefficient and $a$ is the constant for a given material. The work hardening coefficient has been calculated from the slope of the straight line. The value of $n$ is 2.04. According to Onitsch, $n$ is greater than 2 when hardness decreases with the increase of load. It satisfies the prediction of Onitsch [11].

\section{Conclusion}

Single crystals of $p$-hydroxyacetophenone (4hydroxyacetophenone) has been grown by the solution growth technique at $37^{\circ} \mathrm{C}$ using a constant temperature bath $\left( \pm 0.01^{\circ} \mathrm{C}\right)$. The grown crystals have been confirmed using X-ray diffraction studies. The functional groups have been identified by FT-IR analysis. Raman analysis has been made on the as-grown crystal and powdered sample. The aromatic $\mathrm{C}-\mathrm{H}$ bend produces more intense peak in the crystal than in the powder sample. It is the only observation differing from 
the crystal to the powdered sample. It is observed from the thermal gravimetric analysis (TGA) that there is a sharp single stage of weight loss between $140^{\circ} \mathrm{C}$ and $300^{\circ} \mathrm{C}$. There is no loss of water below $125^{\circ} \mathrm{C}$ illustrating the absence of any absorbed water in the sample. Since, the single stage of weight loss starts at $147^{\circ} \mathrm{C}$ without any intermediate stages the compound is expected to evaporate at this temperature. The Vicker's micro-hardness test has been carried out and the work hardening coefficient has been determined.

\section{References}

[1] J. Badan, R. Hierle, A. Perigaud, J. Zyss, NLO properties of organic molecules and polymeric materials, in: D.J. Williams (Ed.), American Chemical Society Symposium Series, Vol. 233, American Chemical Society, Washington, DC, 1993.
[2] D. Chemla, J. Zyss, in: Nonlinear Optical Properties of Organic Materials and Crystals, Academic press, New York, 1987.

[3] T. Sasaki, A. Yokotani, K. Fujioka, T. Yamanka, S. Nakai, C. Yamanaka, NLO organic semiconductors, in: T. Kobayashi (Ed.), Springer Proceedings in Physics, Vol. 36, Springer, Berlin, 1989.

[4] L.I. Zhendeng, W.U. Biachang, S.U. Genbo, J. Crystal Growth 178 (1997) 539.

[5] P. Kerkoc, M. Zgonik, K. Sutter, C. Bosshard, P. Gunter, J. Opt. Soc. Am. B 7 (1990) 313.

[6] S. Chenthamarai, D. Jayaraman, K. Meera, P. Santhanaraghavan, C. Subramanian, G. Bocelli, P. Ramasamy, Crystal Eng. 4 (2001) 37.

[7] N. Bloembergen, J. Nonlinear Opt. Phys. Mater. 15 (1996) 1.

[8] Hou Wenbeo, Yuan Duorong, Xu Dong, Jiang Minhua, J. Crystal Growth 133(1993) 71.

[9] P.N. Prasad, D.J. Williams, Introdution to Nonlinear Optical Effects in Molecules and Polymers, Wiley, New York, 1991.

[10] P.N. Prasad, D.R. Ulrich (Eds.), NLO and Electroactive Polymers, Plenum, New York, 1989.

[11] E.M. Onitsch, Microskope 95 (1950) 12. 